\title{
A Survey on the Management of Chronic Rhinosinusitis in the Korean Rhinologic Society
}

\author{
Gwanghui Ryu, MD and Byoung-Joon Baek, MD, PhD \\ Department of Otorhinolaryngology-Head and Neck Surgery, Soonchunhyang University College of Medicine, Cheonan, Korea
}

Background and Objectives: There are several diagnostic and therapeutic modalities for chronic rhinosinusitis (CRS), but specific guidelines have not been developed for Koreans. This study sought to evaluate and report CRS practice patterns of members of the Korean Rhinologic Society.

Materials and Method: An anonymous survey including 32 items was conducted from August to September 2015. The survey items were categorized into three parts: general information, diagnosis and treatment of CRS, and endoscopic sinus surgery. Results: A total of 267 members participated in this survey. Almost half of the respondents (41.9\%) were in their 40s and $42.7 \%$ had $10-20$ years of experience. A total of $61.8 \%$ were private practitioners, while the rest worked in hospitals (38.2\%). The prevalence rate of acute rhinosinusitis was higher in private clinics compared to hospitals $(\mathrm{p}<0.001)$. Intranasal steroids and saline irrigation were more commonly prescribed among hospital doctors compared to private practitioners $(\mathrm{p}<0.001)$. Amoxicillin/clavulanic acid was the drug of choice for adult and pediatric CRS patients among private practitioners. However, hospital doctors preferred macrolides for adult CRS patients and third-generation cephalosporins for pediatric CRS patients. Most private clinics performed surgery under local anesthesia (90.8\%), while those in a hospital setting preferred general anesthesia (78.4\%). Revision surgery rates were higher in hospitals compared to private clinics $(\mathrm{p}<0.001)$.

Conclusion: There were significant variations in CRS practice patterns between private clinics and hospitals. For effective and standardized diagnosis and management of CRS, appropriate local guidelines are needed.

KEY WORDS: Chronic rhinosinusitis $\cdot$ Medical treatment $\cdot$ Endoscopic sinus surgery $\cdot$ Survey $\cdot$ Guidelines.

\section{INTRODUCTION}

Chronic rhinosinusitis (CRS) is a common otorhinolaryngologic disease, with an annual prevalence of $5-12 \%$. It is characterized by nasal obstruction, purulent rhinorrhea, facial pain, and olfactory dysfunction. In children, CRS symptoms include coughing instead of loss of smell. ${ }^{1)}$ Rhinosinusitis can be classified based on the duration of symptoms, it is defined as acute rhinosinusitis (ARS) when symptoms last for less than 12 weeks, and CRS when the symptoms last for more than 12 weeks. Nasal endoscopy, simple radiography, and computed tomography (CT) are used to diagnose sinusitis. As follow the guideline, CRS can be diagnosed when there is an inflammation (polyp, discharge, or edema) of the nasal cavity by nasal endoscopy, or there is an abnormality in the sinuses by CT scan. ${ }^{1)}$ The first-line treatment for CRS is medical therapy, and endoscopic sinus surgery (ESS) is considered for CRS patients who fail medical therapy.

Although the European (European Position Paper on Rhinosinusitis and Nasal Polyps 2020) and the United States (International Consensus Statement on Allergy and Rhinology: Rhinosinusitis) guidelines are typically used in the diagnosis and treatment of $\mathrm{CRS},{ }^{1,2)}$ the actual diagnosis and

Received: November 2, 2020 / Accepted: November 25, 2020

Address for correspondence: Byoung-Joon Baek, MD, PhD, Department of Otorhinolaryngology-Head and Neck Surgery, Soonchunhyang University College of Medicine, 31 Suncheonhyang 6-gil, Dongnam-gu, Cheonan 31151, Korea

Tel: +82-41-570-2265, Fax: +82-41-579-9022, E-mail: bjbaek@schmc.ac.kr 
treatment in clinical practice vary considerably based on clinician preference and the clinical setting. In particular, the type or duration of CRS medical therapy and surgical treatment varies depending on the surgeon. There are practical challenges in applying overseas treatment guidelines, including treatment costs, medical environment, or differences in antibiotic resistance rates.

From September 2013 to September 2014, Jung et al. ${ }^{3)}$ conducted a questionnaire survey on the ESS practice patterns of the Korean Rhinologic Society members. As pointed out in the previous article, there are no specific CRS guidelines that fit the medical environment in Korea. So far, various questionnaire surveys have been conducted on the treatment patterns of CRS for American Rhinologic Society members. ${ }^{4-9)}$ Similar studies have been conducted in the UK, the Netherlands, and Asia; with each study, reporting varying practice patterns between general practitioners and otorhinolaryngologists. ${ }^{10-12)}$

This study aimed to evaluate and report the CRS practice patterns of the Korean Rhinologic Society members and to compare the practice patterns between private practitioners and hospital employees. The survey included information on diagnosis, medical therapy, and surgical treatment of CRS.

\section{MATERIALS AND METHODS}

An anonymous questionnaire survey including 32 items was conducted from August to September 2015. The 32 survey items were categorized into three parts; general information (age, years of career, and practice type, etc.), diagnosis (image tests, allergy tests, and smell tests), and treatment of CRS, and ESS (Table 1). Questions on medical treatments covered: oral antibiotics (amoxicillin, amoxicillin/clavulanic acid, cephalosporins, macrolides, quinolones, tetracycline, trimethoprim/sulfamethoxazole, and clindamycin), intranasal and oral corticosteroids, intranasal and oral decongestants, antihistamines, mucolytics, antileukotrienes, and saline irrigation. Survey on ESS included: the number of operations, anesthesia methods, surgical methods, postoperative management, and follow-up patterns. This study was approved by the Institutional Review Board (IRB No. 2020-09-018), and the need for informed consent was waived.

\section{Statistical analysis}

Statistical analyses were performed using STATA soft-
Table 1. List of questions used in the survey

\section{General information}

1. What is your age group?

2. How many years of your doctor's career?

3. What is your practice type?

4. What is the total proportion of rhinosinusitis among all your patients?

5. What is the ratio of acute and chronic rhinosinusitis (CRS)?

6. What is the most cost-effective diagnostic tool of rhinosinusitis?

Diagnosis and management of chronic rhinosinusitis

7. How often do you do X-ray or computed tomography (CT) scans to diagnose CRS?

8. How often do you test for allergies or smell for CRS patients?

9. How and what kind of medications do you prescribe for CRS patients?

10. If you prescribe antibiotics, do you carry out a Gram stain and culture test?

11. Which antibiotic is frequently prescribed for adult CRS patients?

12. Which antibiotic is frequently prescribed for pediatric CRS patients?

13. What is the average duration of antibiotic use in adults CRS patients?

14. When do you change antibiotics if the first drug is ineffective?

Endoscopic sinus surgery

15. What are the most common cases of recommending surgery for CRS patients?

16. How many endoscopic sinus surgeries (ESS) do you perform per week?

17. What is the main method of anesthesia for ESS?

18. Do you use the microdebrider for ESS?

19. What is the most common pattern of ESS?

20. What is the percentage of revision surgery among the total number of ESS?

21. Which packing material do you use?

22. When to start saline irrigation after ESS?

23. Which saline solution do you usually prescribe for saline irrigation?

24. How many saline irrigations per day are recommend to the patients after ESS?

25. How long saline irrigations are recommended to the patients after ESS?

26. How long are antibiotics prescribed after ESS?

27. How long are intranasal corticosteroids prescribed after ESS?

28. Do you perform a sinus imaging test after ESS?

29. Do you use an image-guided navigation system for ESS?

30. What is the most intractable symptom after ESS?

31. What is the most common cause of recurrence of CRS after ESS?

32. How do you treat pediatric CRS patients who do not respond to medical treatment? 
ware v14.0 (StataCorp LP, College Station, TX, USA). For each question, the difference between the response rates of the private practitioners and hospital employees (academic and non-academic) was analyzed using the chi-square test or Fisher's exact test. P-value $<0.05$ was considered statistically significant.

\section{RESULTS}

\section{General information}

Of the 267 Korean Rhinologic Society members who answered the questionnaire, $41.9 \%$ were in their $40 \mathrm{~s}$, and $42.7 \%$ had practiced for between 10 and 20 years (Table 2). Among the respondents, there were 165 private practitioners (61.8\%), and 102 (38.2\%) hospital employees, includ-

Table 2. Demographics of the responders

\begin{tabular}{lc}
\hline \multicolumn{1}{c}{ Demographic } & Number $(\%)$ \\
\hline Age & \\
$<40$ years & $75(28.1)$ \\
$40-49$ years & $112(41.9)$ \\
$50-59$ years & $64(24.0)$ \\
$\geq 60$ years & $16(6.0)$ \\
Number of years in practice & \\
$<5$ years & $42(15.7)$ \\
$5-9$ years & $52(19.5)$ \\
10-19 years & $114(42.7)$ \\
$\geq 20$ years & $59(22.1)$ \\
Practice type & \\
Private practice & $165(61.8)$ \\
Hospital employed, nonacademic & $35(13.1)$ \\
Hospital employed, academic & $67(25.1)$ \\
Percentage of rhinosinusitis patients among all patients \\
$<10 \%$ & $30(11.2)$ \\
10-24\% & $115(43.1)$ \\
$25-50 \%$ & $98(36.7)$ \\
$\geq 50 \%$ & $24(9.0)$ \\
The ratio of acute and chronic rhinosinusitis & \\
$9: 1$ & $69(25.9)$ \\
$7: 3$ & $79(29.7)$ \\
$5: 5$ & $35(13.2)$ \\
3:7 & $47(17.7)$ \\
The most cost-effective diagnostic tool & $36(13.5)$ \\
Natient's symptom & $146(55.3)$ \\
Compul & \\
\hline
\end{tabular}

ing academic and non-academic hospitals. Rhinosinusitis patients accounted for $10-50 \%$ of all the patients $(n=213$, $79.8 \%)$ at a ratio of 7:3 and 9:1 for ARS and CRS ( $n=148$, $55.4 \%$ ). The proportion of ARS cases was higher in private practitioners compared to hospital employees $(\mathrm{p}<0.001)$. Cost-effective diagnostic methods were in the order of nasal endoscopy (55.3\%), simple X-ray (16.3\%), patient's symptom (15.2\%), and CT scan (12.9\%), with no statistical differences between the private practitioners and the hospital employees.

\section{Diagnosis and treatment of CRS}

Hospital employees more often performed simple X-ray $(\mathrm{p}=0.020), \mathrm{CT}(\mathrm{p}<0.001)$, allergic tests $(\mathrm{p}<0.001)$, and smell tests $(\mathrm{p}<0.001)$ as compared to the private practitioners ( $\mathrm{Q} 7$ and Q8). There was a significant variation in medical treatment modalities (Q9) between the private practitioners and the hospital employees (Fig. 1). The proportion of respondents who always prescribed intranasal corticosteroids was $7.5 \%$ for the private practitioners and $22.7 \%$ for the hospital employees $(p<0.001)$. Saline irrigation was more often prescribed by the hospital employees $(\mathrm{p}=0.021)$ than by private practitioners. Decongestants $(\mathrm{p}=0.002)$ and $\mathrm{mu}-$ colytics $(\mathrm{p}=0.011)$ prescribing rates were higher among the private practitioners than hospital employees. There was a significant difference in the prescribing rates of antileukotrienes between the two groups ( $\mathrm{p}<0.001)$, with lower rates among the private practitioner as compared to hospital employees, and a lower overall prescribing rate as compared to the other drugs in both groups.

Approximately $30 \%$ of the hospital employees prescribed antibiotics based on the Gram stain and culture results (Q10), however, the number of Gram stain and culture tests was low in both groups $(\mathrm{p}<0.001)$. The antibiotics $(\mathrm{Q} 11,12)$ primarily prescribed for CRS patients were amoxicillin/clavulanic acid (59.3\%) and macrolides (25.9\%). However, hospital employees preferred macrolides (37.0\%), amoxicillin/clavulanic acid (29.0\%), and 3rd generation cephalosporins $(23.0 \%)$ for adult CRS, and amoxicillin/clavulanic acid (54.0\%), and 3rd generation cephalosporins (13.0\%) for pediatric CRS. Both groups preferred antibiotic prescribing period (Q13) of between two and three weeks in adult CRS ( $\mathrm{p}=0.471)$. In the case of antibiotic failure (Q14), switching to a new antibiotic was done after two weeks of use in both groups $(96.3 \%$ and $91.0 \%, \mathrm{p}=0.265)$. 

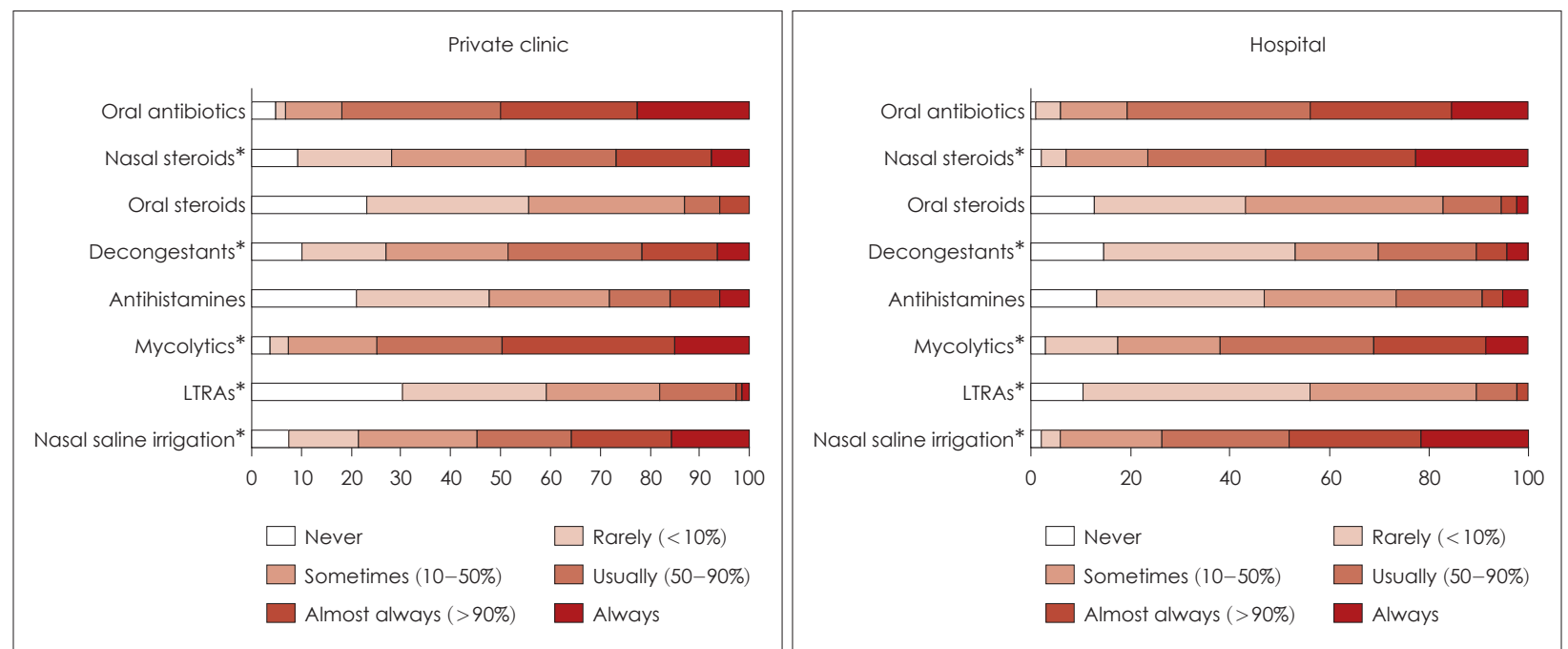

Fig. 1. Comparison of medical treatment modalities for chronic rhinosinusitis between private practitioners and hospital employees. $*: p<0.05$ was considered statistically significant between two groups.

\section{Endoscopic sinus surgery (ESS)}

The most common reason for recommending ESS (Q15) was nasal polyposis $(63.3 \%)$, followed by medically intractable diseases $(26.6 \%)$. The number of ESS performed per week (Q16), was higher among the hospital workers than in the private practitioners $(\mathrm{p}<0.001)$. Private practitioners preferred performing ESS (Q17) under local anesthesia (90.8\%), while the hospital employees preferred general anesthesia (78.4\%). The frequency of microdebrider use during ESS (Q18) was 63.4\% at the private clinics and $92.8 \%$ at the hospitals $(\mathrm{p}<0.001)$. Ethmoidectomy and middle meatal antrostomy were the most common surgical interventions (Q19) among the private practitioners (47.3\%), while ethmoidectomy, frontal sinusotomy, and middle meatal antrostomy were the most common among the hospital workers $(48.5 \%, \mathrm{p}<0.001)$. The percentage of revision surgery out of the total number of ESS (Q20) was higher at the hospitals $(\mathrm{p}<0.001)$ than at the private clinics. The most preferred packing materials after ESS (Q21) among the private practitioners was nonabsorbable packing (78.6\%), while hospital employees preferred absorbable materials $(71.9 \%, \mathrm{p}<0.001)$.

Approximately thirty eight percent of the respondents preferred to start saline irrigation (Q22) one to three days after removal of the packing, $22.8 \%$ preferred to start immediately after removal, and $14.5 \%$ four to five days after removal. Isotonic saline solution was the most commonly used solution (Q23, 98.3\%). The majority of the respondents $(50.9 \%)$ performed saline irrigation twice a day
(Q24), and the preferred duration of saline irrigation (Q25) was between one to three months $(46.3 \%)$. For postoperative medications (Q26 and Q27), 44.6\% of all respondents choose two to four weeks of antibiotics therapy, while $36.8 \%$ choose 1-3 months of intranasal corticosteroids. The majority of the respondents $(88.7 \%)$ did not perform a sinus imaging test after ESS (Q28), while the other respondents choose simple X-ray, ultrasonography, or CT. Image-guided navigation system during surgery (Q29) was rarely utilized at the private clinics (7\%), unlike in hospitals (36.4\%, $\mathrm{p}<0.001)$. The most common intractable symptoms after ESS (Q30) at the private clinics were postnasal drip (42.6\%), while the loss of smell (46.7\%) and postnasal drip (42.4\%) were the most common at the hospitals $(\mathrm{p}<0.001)$. The most common cause of recurrence after ESS (Q31) was in the order of inadequate surgery (27.6\%), lack of postoperative management (27.2\%), asthma and other comorbidities $(22.0 \%)$, at the private clinics, and asthma and other comorbidities (32.8\%), lack of postoperative management (21.9\%), and constitutional factors of the patient (21.3\%) at the hospitals ( $\mathrm{p}=0.002)$. In the case of medical treatment failure in pediatric CRS (Q32), interventions included regular follow-up (50.2\%), adenotonsillectomy (22.5\%), and adenoidectomy $(9.2 \%)$.

\section{DISCUSSION}

In the present study, we conducted an extensive questionnaire survey on the diagnosis and treatment of CRS in 
267 members of the Korean Rhinologic Society. The research aimed at evaluating practical patterns of CRS diagnosis and treatment at the private clinics and hospital, and comparing it with the existing published guidelines. Our study findings showed significant variation in the practice patterns between the private practitioners and hospital employees.

More diagnostic tests such as image studies, allergy tests, and smell tests were performed by the responders working in the hospitals. Intranasal corticosteroids and saline irrigation use were higher at the hospitals as compared to private clinics where decongestants and mucolytics were frequently prescribed. American and European guidelines for CRS recommend intranasal corticosteroids and saline irrigation regardless of nasal polyposis. ${ }^{12)}$ In a previous study investigating the degree of agreement between treatment guideline of CRS and actual practice for members of the American Rhinologic Society, more than $85 \%$ of participants responded 'always or almost always' for saline irrigation and 67\% answered 'always or almost always' for intranasal corticosteroids, indicating a high concordance with the guidelines. ${ }^{4)}$ In this study, the percentage of 'always or almost always' was $36 \%$ for saline irrigation and $27 \%$ for intranasal corticosteroids among the private practitioners, and $48 \%$ for saline irrigation and $53 \%$ for intranasal corticosteroids among the hospital workers. These results showed relatively low concordance with the guidelines.

Amoxicillin/clavulanic acid (59.3\%) were the most preferred antibiotics treatment by the private practitioners, while hospital employees prescribed macrolides (37.0\%) and amoxicillin/clavulanic acid (29.0\%) for adult CRS. The majority of the respondents prescribed antibiotics for a duration of two to four weeks for adult CRS and recommended surgical treatment for patients with nasal polyps or in case of medical treatment failure. European guidelines also suggest that ESS should be considered if there is no improvement after 6 to 12 weeks of appropriate medical therapy. ${ }^{1)}$ Similar to these findings, in a survey of maximal medical therapy for members of the American Rhinologic Society, the majority of the respondents prescribed oral antibiotics and intranasal corticosteroids, and the average duration of antibiotics was 3.1 to 4 weeks. ${ }^{9)}$ Our results were also in agreement with Chinese otolaryngologists who considered ESS in less than three months of maximal medical therapy and preferred intranasal corticosteroids, antibiotics, mucolytics, and saline irrigation. However, they also prescribed oral Chinese herbal medicine. ${ }^{13)}$
Our study findings showed significant variation in anesthesia, uses of microdebrider, surgery patterns, revision surgery rates, and use of navigation systems between the private clinics and hospitals. Inferred from the difference in response rate to these questions, it can be concluded that hospital employees dealt with more severe cases of CRS as compared to private practitioners. These results were similar to a survey conducted for members of the Korean Rhinologic Society in 2013 and 2014. ${ }^{3)}$ In a study on image-guided surgery by the American Rhinologic Society members, the rate of image-guided surgeries increased from $86 \%$ in 2005 to $94.6 \%$ in $2010 .^{8)}$ In the present study, the use of image-guided navigation system was $61.6 \%$ among the hospital workers, which has been increasing since the medical insurance coverage began in 2015. Nonabsorbable packing materials were preferred at the private clinics, whereas absorbable stuff were frequently used at the hospitals. There was no significant difference between the two groups in regard to the number and method of saline irrigation, and postoperative medical treatment, including intranasal corticosteroids and antibiotics.

While our survey was comprehensive, further investigation of refractory CRS and the use of biologics, and the use of biologics is required. Together with additional extensive surveys, and systematic literature reviews, our study provides a framework for developing local guidelines necessary for effective diagnosis and management of CRS.

\section{REFERENCES}

1) Fokkens WJ, Lund VJ, Hopkins C, Hellings PW, Kern R, Reitsma $\mathrm{S}$, et al. European Position Paper on Rhinosinusitis and Nasal Polyps 2020. Rhinology 2020;58:1-464.

2) Orlandi RR, Kingdom TT, Hwang PH, Smith TL, Alt JA, Baroody FM, et al. International Consensus Statement on Allergy and Rhinology: Rhinosinusitis. Int Forum Allergy Rhinol 2016;6 Suppl 1: S22-209.

3) Jung YG, Dhong HJ, Park YJ. Practice Patterns in Endoscopic Sinus Surgery: Survey of the Korean Rhinologic Society. J Rhinol 2015;22: 82-8.

4) Riley CA, Zheng Z, Williams N, Smith TL, Orlandi RR, Tabaee A. Concordance of self-reported practice patterns of American Rhinologic Society members with the International Consensus Statement of Allergy and Rhinology: Rhinosinusitis. Int Forum Allergy Rhinol 2020;10:665-72.

5) Husain Q, Banks C, Bleier BS. Use of off-label steroid irrigations in chronic rhinosinusitis: a survey of the American Rhinologic Society. Int Forum Allergy Rhinol 2020;10:575-6.

6) Smith EJ, Stringer S. Current perioperative practice patterns for minimizing surgical site infection during rhinologic procedures. Int Forum Allergy Rhinol 2014;4:1002-7.

7) González-Castro J, Pascual J, Busquets J. National survey on the 
use of preoperative systemic steroids in endoscopic sinus surgery. Int Forum Allergy Rhinol 2013;3:497-503.

8) Justice JM, Orlandi RR. An update on attitudes and use of imageguided surgery. Int Forum Allergy Rhinol 2012;2:155-9.

9) Dubin MG, Liu C, Lin SY, Senior BA. American Rhinologic Society member survey on "maximal medical therapy" for chronic rhinosinusitis. Am J Rhinol 2007;21:483-8.

10) Vennik J, Eyles C, Thomas M, Hopkins C, Little P, Blackshaw H, et al. Management strategies for chronic rhinosinusitis: a qualitative study of GP and ENT specialist views of current practice in the UK. BMJ Open 2018;8:e22643.
11) Wang DY, Wardani RS, Singh $K$, Thanaviratananich $S$, Vicente $G$, $\mathrm{Xu} \mathrm{G}$, et al. A survey on the management of acute rhinosinusitis among Asian physicians. Rhinology 2011;49:264-71.

12) Hoffmans R, Schermer T, van Weel C, Fokkens W. Management of rhinosinusitis in Dutch general practice. Prim Care Respir J 2011; 20:64-70.

13) Huang Z, Ma J, Sun Y, Zhou B. Maximal Medical Therapy for Chronic Rhinosinusitis: A Survey of Chinese Otolaryngologists. Ear Nose Throat J 2020;99:159-64. 